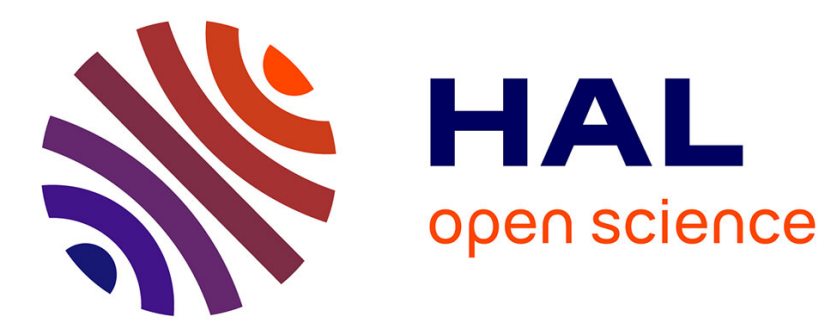

\title{
Acute infections caused by Tropheryma whipplei
}

\author{
Jean-Christophe J.-C. Lagier, Florence Fenollar, Didier Raoult
}

\section{To cite this version:}

Jean-Christophe J.-C. Lagier, Florence Fenollar, Didier Raoult. Acute infections caused by Tropheryma whipplei. Future Microbiology, 2017, 12 (3), pp.247-254. 10.2217/fmb-2017-0178 . hal01521226

\section{HAL Id: hal-01521226 \\ https://hal.science/hal-01521226}

Submitted on 9 May 2018

HAL is a multi-disciplinary open access archive for the deposit and dissemination of scientific research documents, whether they are published or not. The documents may come from teaching and research institutions in France or abroad, or from public or private research centers.
L'archive ouverte pluridisciplinaire HAL, est destinée au dépôt et à la diffusion de documents scientifiques de niveau recherche, publiés ou non, émanant des établissements d'enseignement et de recherche français ou étrangers, des laboratoires publics ou privés. 


\section{SPECIAL REPORT}

For reprint orders, please contact: reprints@futuremedicine.com

\section{Acute infections caused by Tropheryma whipplei}

\author{
Jean-Christophe Lagier' ${ }^{1}$ Florence Fenollar ${ }^{1} \&$ Didier Raoult ${ }^{*, 1}$
}

Tropheryma whipplei is the causative bacterium of Whipple's disease. Its first culture has led to an enlargement of the field of the caused infections. Here, we comprehensively review acute T. whipplei infections. In a cohort study featuring 4000 children, T. whipplei was significantly more common in patients with diarrhea (4\%) than in those without (1.7\%). A case-controlled study highlighted 58 patients suffering from pneumonia with the detection of $T$. whipplei in their bronchoalveolar fluids. Finally, a recent study detected T. whipplei in the blood of 36 febrile patients experiencing pulmonary symptoms in a rural area of Senegal. T. whipplei is definitively an agent of acute gastroenteritis, a cause of nonmalarial fever in Africa, and probably a cause of pulmonary infections.

First draft submitted: 8 September 2016; Accepted for publication: 8 November 2016; Published online: 27 February 2017

Tropheryma whipplei is the causative bacterium of classic Whipple's disease, a chronic and rare infectious disease involving mainly middle-aged Caucasian males [1-3]. Recent epidemiological estimations, however to interpret with caution, have suggested a prevalence of 1.1 per 1 million populations and an annual incidence from one to six per 10 million populations but with strong geographical differences [3]. The typical Whipple's disease clinical involvement associates arthritis followed by diarrhea and weight loss. Whipple's disease is diagnosed by histological analysis of a small-bowel biopsy (positive periodic acid-Schiff staining and/or T. whipplei immunohistochemistry) [2]. Since the first cell culture of T. whipplei in 2000 [4,5], the range of chronic infections or asymptomatic carriage caused by this bacterium, their diagnosis and their management has expanded dramatically [2]. The spectrum of chronic infections caused by T. whipplei includes endocarditis, neurological infections, uveitis, arthritis and osteoarticular infections and, less frequently, isolated adenopathies [2,6]. Moreover, as a result of this culture, the recommended treatment is no longer empirical but evidence-based, on the grounds of both in vitro results and clinical outcomes $[7,8]$. This includes a 1-year combination of doxycycline and hydroxychloroquine followed by a lifetime treatment with doxycycline [7,9-10] because of the lifelong susceptibility of Whipple's disease patients to this bacterium [9]. Some other teams proposed a treatment associating intravenous ceftriaxone followed by a 1-year trimethoprim-sulfamethoxazole regimen [3]. As trimethoprim is inefficient for T. whipplei [2], and as we observed relapses in most of our patients including several cases of resistance to sulfamethoxazole [7,10], we cannot recommend this antibiotic treatment. Finally, T. whipplei stool carriage was also comprehensively described, particularly in sewer workers in France and Germany, in homeless shelters, in healthy individuals in

\section{KEYWORDS}

- gastroenteritis

- pneumonia • Tropheryma

whipplei 


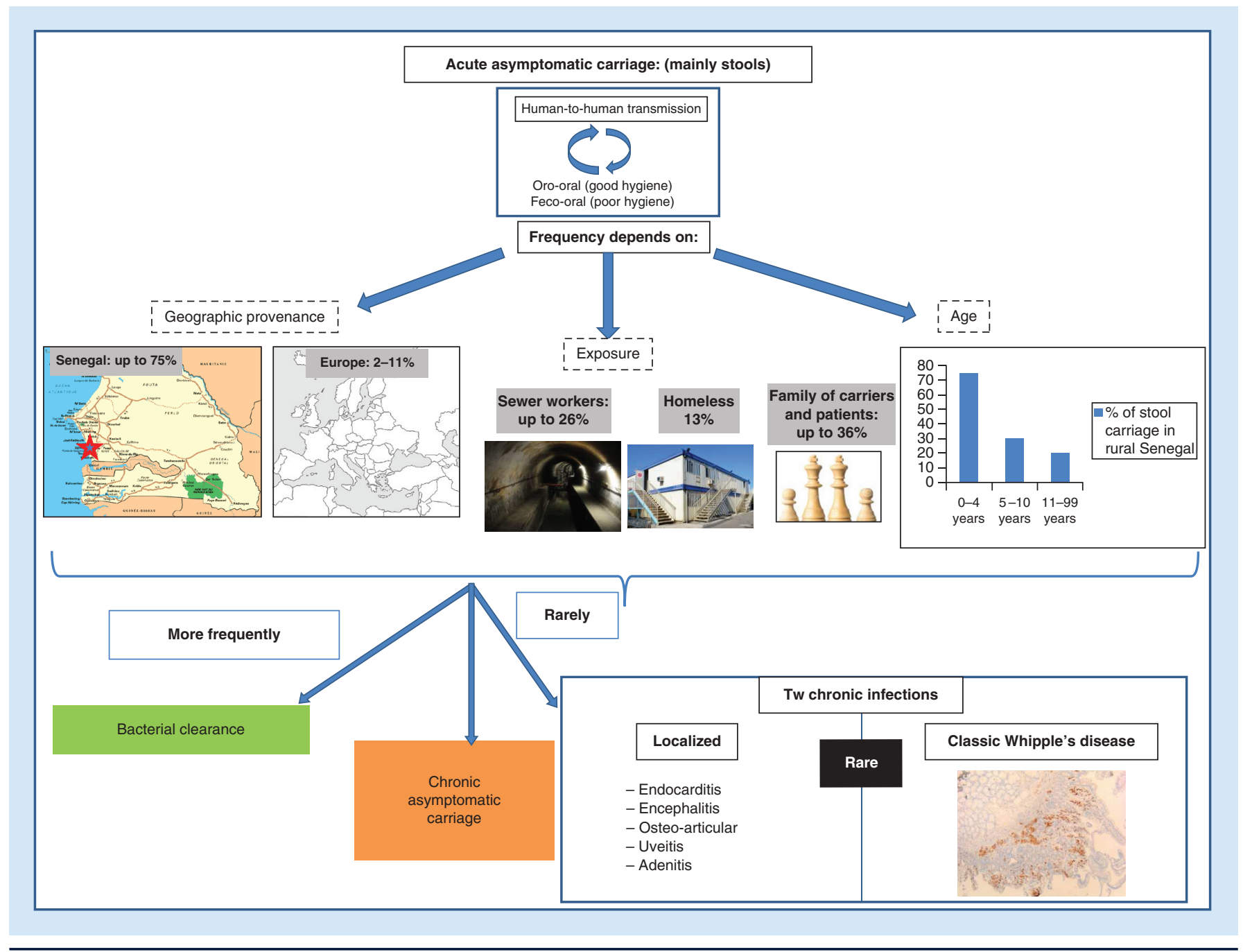

Figure 1. Natural history of Tropheryma whipplei carriage. This summary allows highlighting that T. whipplei acute asymptomatic carriage depends on geographic provenance, age and exposure. In most of the cases, we observe a bacterial clearance although in rare cases, individuals evolve to chronic asymptomatic carriage or to chronic T. whipplei infections.

rural Senegal and in both patients' and carriers' families [2,11-13] (Figure 1). This carriage appears to be worldwide as it has also been observed in Laos and in Ghana [14,15].

However, in addition to these chronic infections and asymptomatic carriage, acute T. whipplei infections may be sometimes encountered [2] (Figure 2). Recently, new data were made available on T. whipplei bacteremia in febrile Senegalese patients, on T. whipplei acute gastroenteritis as well as on T. whipplei pneumonia [16-18]. In this paper, we propose focusing on these acute infections, highlighting the possible natural history of this surprising bacterium. In terms of outlook, we will underline the possible avenues for future research in order to confirm these suggestions.

\section{Tropheryma whipplei bacteremia}

In the first piece of research, from November 2008 to July 2009, a prospective study was performed in two villages in rural Senegal (Dielmo and N'Diop) which have 700 inhabitants in the Sine-Saloum area [19]. Two-hundred and four febrile patients were included in this study. T. whipplei DNA was detected in the blood samples of 13 different patients $(6.4 \%)$ without malaria. The controls were French T. whipplei stool carriers, and none of them had a positive T. whipplei blood sample. Interestingly, although all the patients were febrile, ten (77\%) had a concomitant cough. This symptom as well as sleep disorders were significantly more common in patients with T. whipplei bacteremia than in the 191 febrile patients without T. whipplei 
bacteremia $(\mathrm{p}=0.002$ and $\mathrm{pm}=0.005$, respectively) [19]. No link with feces carriage of T. whipplei was observed [19].

In the second study, which included a control group with healthy people living in the same area, and which was performed in the same two villages, between June 2010 and March 2012, whole-blood finger-prick samples were analyzed from 786 febrile and 385 healthy villagers. Tropheryma whipplei DNA was detected in the blood samples of 36 febrile patients (4.6\%) and one control $(0.25 \%)$ [17]. Seasonality was observed because $100 \%$ of the cases occurred during the rainy season (June-October) but none of the cases occurred during the dry season (November-May). A comprehensive analysis demonstrated that most cases were diagnosed in August 2010, highlighting a probable T. whipplei outbreak. The main clinical manifestations were a headache $(69 \%)$ and a cough $(36 \%)$ with no significant difference between the T. whipplei positive and negative groups. Familial cases were observed, with $75 \%$ of the patients living in a household that had experienced the T. whipplei bacteremia [17]. Finally, because of the lack of samples available, genotyping has been only established for eight patients. These partial data lead us to suspect the presence of a potential but not confirmed new epidemic clone. Despite the low number of strains that have been genotyped, the authors suspected that an epidemic clone was present. Interestingly, the local baker lived in the household where the prevalence of T. whipplei was the highest. Since the departure of this baker and his family, no outbreaks were observed and a concomitant decrease of T. whipplei prevalence was also noted [17].

In another African country, Gabon, the presence of T. whipplei in blood samples of febrile patients was investigated between 2014 and 2015. T. whipplei DNA was detected with a prevalence of $0.2 \%$ in the 410 febrile patients living in both rural and urban areas [20]. Tropheryma whipplei was detected only in febrile patients and not in controls. In conclusion, the real prevalence of T. whipplei bacteremia is currently difficult to

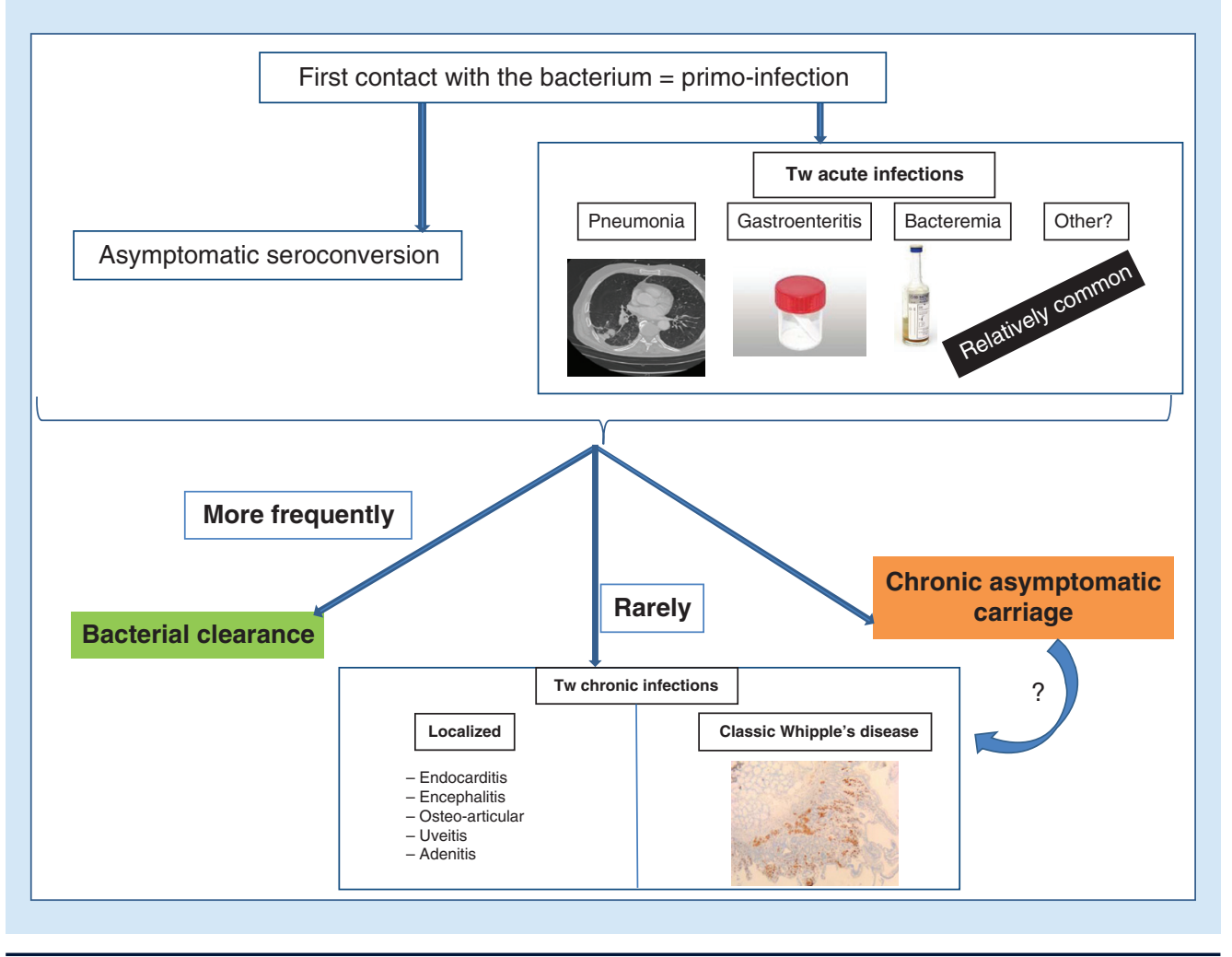

Figure 2. Natural history of Tropheryma whipplei infections. This summary highlights that T. whipplei acute infection consists more frequently of an asymptomatic seroconversion and in rare cases in T. whipplei acute infections. In most of the cases, we observe a bacterial clearance although in rare cases, individuals evolve to chronic asymptomatic carriage or to chronic T. whipplei infections. 
estimate, but these recent large-scale studies confirm the role of T. whipplei in febrile nonmalarial episodes and indicate that it may depend on the epidemic situation.

\section{Gastroenteritis}

A variety of strong data have been accumulated over the years pointing to a link between acute diarrhea and T. whipplei. A first major study was conducted in France looking at the presence of T. whipplei DNA in stool samples of children aged between two and four. Tropheryma whipple $i$ was detected in 36 of the 241 samples tested (15\%) from children suffering from gastroenteritis, while none of the 47 controls tested were positive $(p=0.008)$. The rate of positive samples was comparable between 2006 and 2008 and no seasonal variation was observed [21]. Most of the patients had a high bacterial load (>104/g of stool), comparable to that of patients suffering from Whipple's disease. A third of the children were coinfected with another pathogen. Despite the large heterogeneity of the strains, evidence of clonal circulation was observed. Experimental model, demonstrating that orally administered T. whipplei may induce diarrhea in mice, has confirmed Koch's postulates that T. whipplei is a bacteria which causes gastroenteritis [22] .

In a hospital-based case-control study performed in rural Ghana on 534 stool samples of children aged between 2 months and 15 years, T. whipplei DNA was detected in $27.5 \%$ of the samples. Overall, no difference between cases and controls was observed, although in the children under the age of one, T. whipplei was more likely to be detected in patients $(23.3 \%)$ than in the controls $(11.4 \%)$ suggesting a possible role in gastroenteritis in young children in rural Africa [15]. In rural Senegal, T. whipplei was also more significantly detected in stool samples of patients with diarrhea $(25 / 51,49 \%)$ compared with asymptomatic individuals $(139 / 446,31.2 \% ; \mathrm{p}=0.015)$ and only in patients under 11 years old [11].

In a subsequent study performed between 2009 and 2013, the link between T. whipplei and acute diarrhea was investigated in a larger cohort of children [18]. A total of 3796 rectal swabs were collected from the emergency departments of hospitals in Marseille, France and were tested for T. whipplei. When stool samples were also available for children with diarrhea, tests were carried out to detect Salmonella, Shigella, Campylobacter and Yersinia species, Escherichia coli 0157:H7, Aeromonas species as well as the presence of rotavirus, adenovirus, norovirus and astrovirus. Of the 3796 children included, 555 suffered from diarrhea. Among them, 22 (4\%) were positive for T. whipplei and a significant difference emerged when compared with the 56 positive samples among the 3241 nondiarrheic children tested $(1.7 \%, \mathrm{p}=0.001)$. T. whipplei was occasionally detected in association with adenovirus and rotavirus. Monomicrobial detection of T. whipplei was observed in eight cases, whereas monomicrobial detection of Salmonella spp. was only observed in four cases. The incidence of diarrhea associated with T. whipplei varied from year to year, from $2 \%$ in 2012 [18], 15\% in 2006-2008 [21], to $16 \%$ in 2009 [18]. In this large cohort, the seasonal circulation of $T$. whipplei was mainly observed during autumn [18].

Among travelers to Senegal, 59 individuals returned stool samples both before and after travel. Of them, two patients (3.4\%) who had no diarrhea were negative for T. whipplei before travel and positive after travel, demonstrating that stool carriage was acquired after travel in a tropical area [23]. In another study performed on 129 pilgrims in Saudi Arabia which analyzed the presence of T. whipplei DNA on rectal swabs before and after Hajj and during episodes of diarrhea, it was revealed that two of nine pilgrims with diarrhea during their pilgrimage were positive for T. whipplei [24]. Further studies exploring this issue in larger cohorts of travelers, including stays in rural area, would contribute toward a better understanding of the potential role of T. whipplei in traveler's diarrhea.

\section{Lung involvement \\ - Pneumonia}

Lung involvement is a rare but classic manifestation of Whipple's disease [1,25], with an isolated cough sometimes being the first clinical involvement revealing the disease [26]. In terms of acute pneumonia, T. whipplei was first detected in 2007 by Harris et al. from bronchoalveolar (BAL) fluid when they were investigating the microbiota of children with cystic fibrosis [27]. Tropheryma whipplei was detected in a control from a previously healthy child suffering from acute interstitial pneumonia [27]. A study performed on patients hospitalized with pneumonia in an intensive care unit in Marseille, France, detected T. whipplei DNA in six of the 210 (3\%) BAL fluids tested [28]. Of these six patients, three suffered from aspiration pneumonia, two from a ventilator-associated pneumonia and one 
from a community-acquired pneumonia [28]. Interestingly, for this last patient, T. whipplei was the only microorganism identified and half of the six patients were immunocompromised. In addition to this study, a case of T. whipplei pneumonia has been reported in a 24-year-old Senegalese HIV-infected patient with a CD4positive cell count of $269 / \mathrm{mm}^{3}$ [29]. A chest CT scan revealed a right upper lobe pneumonia as well as bilateral alveolar condensation. Blood culture, Legionella pneumophila and Streptococcus pneumoniae urinary antigens were negative, and analysis of BAL fluid for Mycobacterium tuberculosis, Pneumocystis jirovecii, Aspergillus sp., cytomegalovirus and herpes virus was also negative. The pure T. whipplei culture from the BAL fluid of a 70-year-old patient suffering from pneumonia was performed for the first time in 2012, for supporting the role of this bacterium in acute respiratory infections [30]. This patient suffered from 3 weeks of nocturnal sweats with fever and dyspnea. Histological examination of the duodenal biopsy sample was negative (periodic acid-Schiff staining and immunohistochemistry) and, unfortunately, the sample was not available for histological analysis focusing on T. whipplei involvement.

Recently, a case-control study of 1438 BAL tested for T. whipplei by qPCR, included 88 patients that were positive and 88 controls matched for aged, sex and hospitalization unit [16]. Of them, 58 patients suffered from pneumonia $(66 \%)$ in the T. whipplei group, while $72 / 88$ $(81 \%)$ in the control group had pneumonia. Tropheryma whipplei was significantly associated with aspiration pneumonia $(18 / 88$ cases in comparison with $6 / 88$ controls, $\mathrm{p}=0.01$ ) and T. whipplei was detected as the sole pathogen in nine additional patients [16]. Overall, immunocompromised status was no different between the T. whipplei group and patients but, despite a low number of patients, T. whipplei was more likely to emerge in AIDS patients with a low CD4 level as well as in patients receiving TNF- $\alpha$ inhibitor treatment. Interestingly, regarding a potentially similar susceptibility, $P$. jirovecii was more frequently associated with the T. whipplei group compared with control group [16].

\section{- Other lung \& airways involvements associated with Tropheryma whipplei}

Alongside acute pneumonia, several studies have reported the presence of T. whipplei in the airways either as probable colonization or as another clinical involvement for which their potential implication continues to be unknown. Recently, a metagenomic study comparing both lung and oral microbiota from 64 healthy subjects revealed a high T. whipplei prevalence of $26 \%$ in BAL lavage samples but not in oral washes [31]. The increased presence of T. whipplei in BAL compared with washes was also observed in another larger study which included HIVinfected and HIV-negative individuals [32]. In addition, a higher prevalence of T. whipplei lung colonization in asymptomatic HIV-infected individuals was observed in comparison with a control group. Moreover, a decrease in the T. whipplei bacterial load was observed after initiation of antiretroviral therapy [33].

In addition, a recent study demonstrated the potential implications of this bacterium on patients with poorly controlled asthma [34]. Thirty patients were included and analysis of the sputum microbiome was performed by $16 \mathrm{SrRNA}$ pyrosequencing and T. whipplei $\mathrm{qPCR}$ for confirmation of the results. Overall, 12 (40\%) of the patients were detected with T. whipplei DNA. In another metagenomic study analyzing the lung microbiome from BAL fluid samples, one of the 29 patients tested who had cortico-resistant asthma, had T. whipplei as a unique microorganism in his airways [35]. Additional studies are needed to prove a relationship between T. whipplei and asthma.

\section{- Place of these acute infections in the natural history of Tropheryma whipplei}

Epidemiological studies on T. whipplei carriage which were recently performed demonstrated human-to-human T. whipplei transmission $[11,12,14,36]$. The prevalence of this carriage depends upon geographical provenance, mode of exposure and age (Figure 1) $[2,3]$. Most patients fully clear the bacterium but, rarely, some patients have developed chronic carriage or a chronic T. whipplei infection (Figure 1). Of the chronic carriers, some appear to be protected against reinfection because they have a strong immune response, while others with a low immune response may be recolonized by another $T$. whipplei strain [37]. In addition, these 175 acute T. whipplei infections (Table 1 \& Figure 2), correspond to another mode of primo-infection. This pointed toward strong evidence on the putative pathway of the natural history of T. whipplei, implying that most patients developing the relatively frequent acute T. whipplei infection could spontaneously clear the bacteria, although a tiny 


\begin{tabular}{|c|c|c|c|c|c|c|}
\hline $\begin{array}{l}\text { Type of } \\
\text { involvement }\end{array}$ & $\begin{array}{l}\text { Type of } \\
\text { studies }\end{array}$ & Place of inclusion & Countries & $\begin{array}{l}\text { Years of } \\
\text { inclusion }\end{array}$ & Patients (n) & Ref. \\
\hline \multicolumn{7}{|l|}{ Pneumonia } \\
\hline & Cohort & Control & USA & 2006 & 1 & [27] \\
\hline & Cohort & Hospitalized in intensive care units & France & 2007-2008 & 6 & [28] \\
\hline & Case report & AIDS-infected outpatient & France & 2013 & 1 & [29] \\
\hline & Case control & Hospitalized in university hospital & France & $2013-2014$ & 57 & {$[16]$} \\
\hline \multicolumn{7}{|l|}{ Gastroenteritis } \\
\hline & Cohort & University hospital for children & France & $2006-2008$ & 22 & [21] \\
\hline & Cohort & Emergency departments of university hospitals for children & France & $2009-2013$ & 36 & {$[18]$} \\
\hline & Cohort & Hajj pilgrims & Saudi Arabia & 2013 & 2 & [24] \\
\hline \multicolumn{7}{|l|}{ Bacteremia } \\
\hline & Cohort & Febrile outpatients & Senegal & $2008-2009$ & 13 & {$[19]$} \\
\hline & Cohort & Febrile outpatients & Senegal & 2010-2012 & 36 & {$[17]$} \\
\hline & Cohort & Febrile outpatients & Gabon & 2013-2014 & 1 & [20] \\
\hline \multicolumn{3}{|c|}{ Total no. of patients } & & & 175 & \\
\hline
\end{tabular}

proportion of them developed a chronic infection due to an undetermined genetic predisposition, while another tiny proportion could develop chronic asymptomatic carriage [2,37] (Figure 2).

\section{Conclusion \& future perspective}

T. whipplei, the causative agent of Whipple's disease, can be involved in acute infections as gastroenteritis or pneumonia and is a cause of nonmalarial fever in Africa.

Most of the studies discussed in this paper were performed in our reference laboratory. We are confident in the results because these studies were conducted on large populations including controls, because the detection of $T$. whipplei was considered after at least two positive specific qPCR tests, and because stringent protocols, including positive and negative controls, were used [38]. Nevertheless, our results are supported by the fact that other teams have also detected T. whipplei using different techniques whether in gastroenteritis [15], pneumonia and lung carriage, or other airway involvements $[27,33,35,39]$. Interestingly, a key issue to underline remains interpretation of the results of such studies when a same pathogen is associated with both colonization and infections. Indeed, we consider that T. whipplei is one of the main significant examples of the absolute need to use controls in the study of epidemiological acute infectious diseases [40]. Tropheryma whipplei is a proof of this concept, whether on stool samples or in BAL fluids samples, or in blood samples where this bacterium was sometimes detected in totally asymptomatic individuals [16-18].

The involvement of T. whipplei in acute infections is definitively based on strong evidence, at least in cases of acute gastroenteritis for which there is strong epidemiological and clinical data as well as an experimental model [18,21,22] . Further larger studies will be needed to support the hypothesis that T. whipplei is a causative agent of traveler's diarrhea [23,24]. Regarding pneumonia, the recent case-controlled study provides definitive clinical and epidemiological evidence [16]. Moreover, the immunocompromised status of patients with T. whipplei pneumonia has not yet been thoroughly elucidated and larger specific studies could be designed [16]. Further study in countries other than Senegal continues to be needed to confirm that T. whipplei is a causative agent of nonmalarial fever in rural Africa. Finally, future large studies will allow to better estimate the frequency of acute infections caused by $T$. whipplei.

\section{Financial \& competing interests disclosure}

The translation assistance by TradOnline was funded by the Centre National de Recherche Scientifique. The authors have no other relevant affliations or financial involvement with any organization or entity with a financial interest in or financial conflict with the subject matter or materials discussed in the manuscript apart from those disclosed.

The authors thank TradOnline (http://www.tradonline. fr/en/) for providing English corrections. 


\section{EXECUTIVE SUMMARY}

- Tropheryma whipplei is the causative agent of Whipple's disease, a rare chronic infectious disease.

- The first culture of T. whipplei, performed in 2000, has improved knowledge of the infections caused by this bacterium.

- Tropheryma whipplei has also been reported as an agent of acute infections including gastroenteritis, bacteremia and acute pneumonia.

- Epidemiological and clinical data as well as experimental model have demonstrated that T. whipplei is an agent of acute gastroenteritis.

- Tropheryma whipplei DNA was detected in the blood samples of 36 febrile patients (4.6\%) and only one control (0.25\%) is a cause of nonmalarial fever in Africa.

- In acute pneumonia, T. whipplei, sometimes detected as sole pathogen, is significantly associated with aspiration pneumonia and is more likely detected in AIDS patients with a low CD4 level and in patients treated by TNF- $\alpha$ inhibitors.

\section{References}

Papers of special note have been highlighted as:

- of interest; $\bullet$ of considerable interest

1 Lagier JC, Lepidi H, Raoult D, Fenollar F. Systemic Tropheryma whipplei: clinical presentation of 142 patients with infections diagnosed or confirmed in a reference center. Medicine (Baltimore) 89(5), 337-345 (2010).

2 Fenollar F, Lagier JC, Raoult D. Tropheryma whipplei and Whipple's disease. J. Infect. 69(2), 103-112 (2014).

3 Marth T, Moos V, Muller C, Biagi F, Schneider T. Tropheryma whipplei infection and Whipple's disease. Lancet Infect. Dis. 16(3), e13-e22 (2016).

4 Raoult D, Birg ML, La SB et al. Cultivation of the bacillus of Whipple's disease. N. Engl. J. Med. 342(9), 620-625 (2000).

-. The first culture of Tropheryma whipplei has allowed to revolutionize the natural history knowledge of this bacterium.

5 Lagier JC, Edouard S, Pagnier I, Mediannikov O, Drancourt M, Raoult D. Current and past strategies for bacterial culture in clinical microbiology. Clin. Microbiol. Rev. 28(1), 208-236 (2015).

6 Fenollar F, Celard M, Lagier JC, Lepidi H, Fournier PE, Raoult D. Tropheryma whipplei endocarditis. Emerg. Infect. Dis. 19(11), 1721-1730 (2013).

7 Lagier JC, Fenollar F, Lepidi H, Giorgi R, Million M, Raoult D. Treatment of classic Whipple's disease: from in vitro results to clinical outcome. J. Antimicrob. Chemother. 69(1), 219-227 (2014).

8 Lagier JC, Fenollar F, Lepidi H, Liozon E, Raoult D. Successful treatment of immune reconstitution inflammatory syndrome in Whipple's disease using thalidomide. J. Infect. 60(1), 79-82 (2010).
9 Lagier JC, Fenollar F, Lepidi H, Raoult D. Evidence of lifetime susceptibility to Tropheryma whipplei in patients with Whipple's disease. J. Antimicrob. Chemother. 66(5), 1188-1189 (2011).

10 Lagier JC, Fenollar F, Lepidi H, Raoult D. Failure and relapse after treatment with trimethoprim/sulfamethoxazole in classic Whipple's disease. J. Antimicrob. Chemother. 65(9), 2005-2012 (2010).

11 Keita AK, Bassene H, Tall A et al. Tropheryma whipplei: a common bacterium in rural Senegal. PLoS Negl. Trop. Dis. 5(12), e1403 (2011).

12 Keita AK, Brouqui P, Badiaga S et al. Tropheryma whipplei prevalence strongly suggests human transmission in homeless shelters. Int. J. Infect. Dis. 17(1), e67-e68 (2013).

13 Fenollar F, Keita AK, Buffet S, Raoult D. Intrafamilial circulation of Tropheryma whipplei, France. Emerg. Infect. Dis. 18(6), 949-955 (2012).

14 Keita AK, Dubot-Peres A, Phommasone K et al. High prevalence of Tropheryma whipplei in Lao kindergarten children. PLoS Negl. Trop. Dis. 9(2), e0003538 (2015).

15 Vinnemeier CD, Klupp EM, Krumkamp R et al. Tropheryma whipplei in children with diarrhoea in rural Ghana. Clin. Microbiol. Infect. 22(1), 65-73 (2016).

16 Lagier JC, Papazian L, Fenollar F et al. Tropheryma whipplei DNA in bronchoalveolar lavage samples: a case control study. Clin. Microbiol. Infect.22(10), 875-879 (2016).

-. A case-control study demonstrating the role of T. whipplei in pneumonia.

17 Bassene H, Mediannikov O, Socolovschi C et al. Tropheryma whipplei as a cause of epidemic fever, Senegal, 2010-2012. Emerg. Infect. Dis. 22(7), 1229-1334 (2016).

18 Fenollar F, Minodier P, Boutin A et al. Tropheryma whipplei associated with diarrhea in young children. Clin. Microbiol. Infect. 22(10), 869-874 (2016).

19 Fenollar F, Mediannikov O, Socolovschi C et al. Tropheryma whipplei bacteremia during fever in rural West Africa. Clin. Infect. Dis. 51(5), 515-521 (2010).

20 Mourembou G, Nzondo SM, NdjoyiMbiguino A et al. Cocirculation of plasmodium and bacterial deoxyribonucleic acids in blood of febrile and afebrile children from urban and rural areas in Gabon. Am. J. Trop. Med. Hyg. 95(1), 123-132 (2016).

21 Raoult D, Fenollar F, Rolain JM et al. Tropheryma whipplei in children with gastroenteritis. Emerg. Infect. Dis. 16(5), 776-782 (2010).

22 Al MK, Malou N, Mege JL, Raoult D, Desnues B. An experimental mouse model to establish Tropheryma whipplei as a diarrheal agent. J. Infect. Dis. 204(1), 44-50 (2011).

23 Gautret P, Lagier JC, Benkouiten S, Fenollar F, Raoult D, Brouqui P. Does Tropheryma whipplei contribute to travelers' diarrhea?: a PCR analysis of paired stool samples in French travelers to Senegal. Travel Med. Infect. Dis. 12(3), 264-267 (2014).

24 Gautret P, Benkouiten S, Parola P, Brouqui P, Memish Z, Raoult D. Occurrence of Tropheryma whipplei during diarrhea in Hajj pilgrims: a PCR analysis of paired rectal swabs. Travel Med. Infect. Dis. 12(5), 481-484 (2014).

25 Urbanski G, Rivereau P, Artru L, Fenollar F, Raoult D, Puechal X. Whipple disease revealed by lung involvement: a case report and literature review. Chest 141(6), 1595-1598 (2012). 
26 Damaraju D, Steiner T, Wade J, Gin K FitzGerald JM. Clinical problem-solving. A surprising cause of chronic cough. $N$. Engl. J. Med. 373(6), 561-566 (2015).

27 Harris JK, De Groote MA, Sagel SD et al. Molecular identification of bacteria in bronchoalveolar lavage fluid from children with cystic fibrosis. Proc. Natl Acad. Sci. USA 104(51), 20529-20533 (2007).

28 Bousbia S, Papazian L, Auffray JP et al. Tropheryma whipplei in patients with pneumonia. Emerg. Infect. Dis. 16(2), 258-263 (2010).

29 Stein A, Doutchi M, Fenollar F, Raoult D. Tropheryma whipplei pneumonia in a patient with HIV2 infection. Am. J. Respir. Crit. Care Med. 188(8), 1036-1037 (2013).

30 Fenollar F, Ponge T, La SB, Lagier JC, Lefebvre M, Raoult D. First isolation of Tropheryma whipplei from bronchoalveolar fluid and clinical implications. J. Infect. 65(3), 275-278 (2012).
31 Morris A, Beck JM, Schloss PD et al. Comparison of the respiratory microbiome in healthy nonsmokers and smokers. Am. J. Respir. Crit. Care Med. 187(10), 1067-1075 (2013).

32 Beck JM, Schloss PD, Venkataraman A et al. Multicenter comparison of lung and oral microbiomes of HIV-infected and HIVuninfected individuals. Am. J. Respir. Crit. Care Med. 192(11), 1335-1344 (2015).

33 Lozupone C, Cota-Gomez A, Palmer BE et al. Widespread colonization of the lung by Tropheryma whipplei in HIV infection. Am. J. Respir. Crit. Care Med. 187(10), 1110-1117 (2013)

34 Simpson JL, Daly J, Baines KJ et al. Airway dysbiosis: Haemophilus influenzae and Tropheryma in poorly controlled asthma. Eur. Respir. J. 47(3), 792-800 (2016).

35 Goleva E, Jackson LP, Harris JK et al. The effects of airway microbiome on corticosteroid responsiveness in asthma. Am. J. Respir. Crit. Care Med. 188(10), 1193-1201 (2013).
36 Fenollar F, Trani M, Davoust B et al. Prevalence of asymptomatic Tropheryma whipplei carriage among humans and nonhuman primates. J. Infect. Dis. 197(6), 880-887 (2008)

37 Fenollar F, Marth T, Lagier JC, Angelakis E, Raoult D. Sewage workers with low antibody responses may be colonized successively by several Tropheryma whipplei strains. Int. J. Infect. Dis. 35, 51-55 (2015).

38 Edouard S, Fenollar F, Raoult D. The rise of Tropheryma whipplei: a 12-year retrospective study of PCR diagnoses in our reference center. J. Clin. Microbiol. 50 (12), 3917-3920 (2012).

39 Qin S, Clausen E, Lucht L et al. Presence of Tropheryma whipplei in different body sites in a cohort of healthy subjects. Am. J. Respir. Crit. Care Med. 194(2), 243-245 (2016).

40 Dubourg G, Fenollar F. Epidemiologic studies need asymptomatic controls. Clin. Microbiol. Infect. 21(8), e51-e52 (2015). 